\title{
Effects of diurnal variation and prolonged refractoriness on repeated measurements of airways responsiveness to methacholine
}

\author{
J R Beach, S C Stenton, M J Connolly, E H Walters, ${ }^{*}$ D J Hendrick
}

\begin{abstract}
Background - A number of studies have suggested that diurnal variation in airways responsiveness underlies the circadian rhythm of ventilatory function in asthma. Measurements of airways responsiveness are therefore often performed at standardised times in order to avoid this possible effect, but this is not practical for epidemiological studies. Refractoriness to methacholine has also been reported and this, too, could confound the results of methacholine tests repeated over short intervals. This investigation was carried out to evaluate the possible magnitude of diurnal variation and refractoriness in repeated measures of airways responsiveness to methacholine.
\end{abstract}

Methods - To investigate diurnal variation in airways responsiveness, 24 asthmatic subjects aged 18-45 underwent five methacholine tests over three days which were not necessarily consecutive: day 1 at 08:00 hours; day 2 at 08:00 hours, 14:00 hours, 20:00 hours; day 3 at 20:00 hours. To investigate refractoriness a retrospective analysis was undertaken of all paired methacholine tests performed in individuais within our unit between 1984 and 1990 where there had been no intervention likely to affect the results.

Results - The first investigation revealed no diurnal change in airways responsiveness although there was a change in $\mathrm{FEV}_{1}$. Mean $\mathrm{PD}_{20}$ did, however, increase 1.57 fold from 08:00 hours on day 1 to 08:00 hours on day 2 for subjects studied on consecutive days. The second investigation confirmed that a test interval of up to 24 hours (but not of 48 or more hours) was associated with a refractory index $\left(\mathbf{P D}_{20}\right.$ test $\mathbf{2}_{\mathbf{P D}} \mathbf{2 0}_{20}$ test 1$)$ of $>1$.

Conclusions - No diurnal variation in airways responsiveness was detected for measurements made between 08:00 hours and 20:00 hours, but an interval between successive tests of up to 24 hours was associated with refractoriness. Diurnal variation is not likely to exert an important confounding effect on methacholine tests carried out between 08:00 hours and 20:00 hours, but confounding could result from refractoriness if tests are repeated at intervals up to 24 hours.

(Thorax 1995;50:235-239)

Keywords: methacholine, bronchial hyperreactivity, circadian rhythm, refractory period.
Measurements of airways responsiveness have become widely used in asthma research. They may be used to quantify the beneficial (and adverse) effects of novel pharmaceutical agents, and the putative aetiological roles of occupational and environmental exposures. In epidemiological investigations they may provide objective evidence of asthma prevalence.

Diurnal variation in airways responsiveness to histamine and other bronchoconstrictor agents has been described, although its magnitude is said to be relatively small compared with the variation in ventilatory function which is seen in many asthmatics. ${ }^{1-7}$ Some studies have not confirmed its existence, while others have found only equivocal evidence of it. ${ }^{267}$ Its importance as a potential confounder of epidemiological investigations which require measurements to be made throughout the working day has not been fully investigated, while in laboratory studies most investigators circumvent the problem by using a standardised time for tests of airways responsiveness.

The primary aim of this study was to investigate the practical importance of a circadian rhythm in airways responsiveness in order to evaluate its possible confounding effect if methacholine tests are performed at different times throughout the working day for epidemiological investigations. When the initial investigation suggested, instead, the possibility of refractoriness to methacholine lasting up to 24 hours, this was investigated further by a retrospective analysis of all previous paired methacholine challenge tests in individual subjects performed within our unit during the period 1984-90.

\section{Methods}

INVESTIGATION 1: SERIAL MEASUREMENTS OF AIRWAYS RESPONSIVENESS OVER THREE DAYS Twenty four asthmatic subjects (18-45 years, 12 women) with measurable airways responsiveness were recruited. All gave informed consent and the study was approved by the local ethics committee. Subjects with an exacerbation of asthma, change in treatment within the preceding six weeks, or forced expiratory volume in one second $\left(\mathrm{FEV}_{1}\right)$ of less than $60 \%$ of predicted were excluded. Inhaled corticosteroids and caffeine containing drinks were withheld for 12 hours before each test, as were inhaled $\beta$ agonists other than those prescribed to reverse the methacholine induced bronchoconstriction following each test. No subjects were taking inhaled long acting $\beta_{2}$ 
agonists, anticholinergics, disodium cromoglycate, oral theophyllines, antihistamines, or oral $\beta_{2}$ agonists at the time.

Doubling cumulative doses of methacholine from $3 \cdot 125 \mu \mathrm{g}$ to $6400 \mu \mathrm{g}$ were administered using a dosimeter technique at five minute intervals until a $>20 \%$ decrement in $\mathrm{FEV}_{1}$ was obtained.$^{8}$ For subjects with low levels of airways responsiveness a shortened protocol was used omitting the lower doses of methacholine. This followed more closely our usual practice in epidemiological studies. ${ }^{9}$ The dose causing a $20 \%$ decrement in $\mathrm{FEV}_{1}\left(\mathrm{PD}_{20}\right)$ was then estimated by linear interpolation from a plot of the cumulative dose of methacholine against percentage fall in $\mathrm{FEV}_{1}$. Providing the cumulative dose is used in calculating $\mathrm{PD}_{20}$ the shortened protocol produces the same result. The dose-response slope (percentage fall after the final dose of methacholine divided by the dose) was also calculated. ${ }^{111}$ Although not strictly interchangeable, a dose-response slope of $1 \%$ decrement in $\mathrm{FEV}_{1}$ per $\mu \mathrm{g}$ methacholine would approximate to a $\mathrm{PD}_{20}$ of $20 \mu \mathrm{g}$, and a dose-response slope of $0.025 \% / \mu \mathrm{g}$ to a $\mathrm{PD}_{20}$ of $800 \mu \mathrm{g}$. Salbutamol $200 \mu \mathrm{g}$ was administered at the end of each test to reverse any bronchoconstriction. Each subject underwent five methacholine tests over three study days. Tests were performed at 08:00 hours on day 1 , at 08:00 hours, 14:00 hours, and 20:00 hours on day 2 , and at 20:00 hours on day 3. Although it was not stipulated in the protocol that test days should be consecutive, it was often convenient for the subjects if they were. Thus test days were consecutive for 19 subjects and spanned a maximum period of 30 days for the remaining five subjects. For the latter both test days 1 and 2 and test days 2 and 3 were nonconsecutive.

INVESTIGATION 2: REVIEW OF PREVIOUS MEASUREMENTS OF AIRWAYS RESPONSIVENESS All data from subjects undergoing two methacholine tests within the previous six years

Table $1 P D_{20}$ ( $\mu g$ methacholine) measurements for all 24 subjects at each measurement point (investigation 1)

\begin{tabular}{rccccl}
\hline $\begin{array}{l}\text { 08:00 hours } \\
\text { (day 1) }\end{array}$ & $\begin{array}{l}\text { 08:00 hours } \\
(\text { day 2) }\end{array}$ & $\begin{array}{c}14: 00 \text { hours } \\
(\text { day } 2)\end{array}$ & $\begin{array}{l}20: 00 \text { hours } \\
\text { (day 3) }\end{array}$ & $\begin{array}{l}20: 00 \text { hours } \\
\text { (day } 3)\end{array}$ & $\begin{array}{l}\text { Tests on } \\
\text { consecutive days }\end{array}$ \\
\hline 25 & 70 & 140 & 200 & 65 & Yes \\
30 & 8 & 3 & 7 & 16 & No \\
32 & 20 & 18 & 30 & 25 & Yes \\
2500 & $6400^{*}$ & 5000 & $6400^{*}$ & $6400^{*}$ & Yes \\
130 & 210 & 235 & 105 & 115 & Yes \\
26 & 72 & 69 & 21 & 13 & No \\
250 & 520 & 1100 & 650 & 408 & No \\
116 & 146 & 112 & 82 & 103 & Yes \\
19 & 26 & 34 & 20 & 16 & Yes \\
37 & 36 & 40 & 110 & 283 & Yes \\
100 & 70 & 160 & 50 & 125 & Yes \\
115 & 290 & 275 & 1400 & 970 & Yes \\
400 & 1350 & $6400^{*}$ & $6400^{*}$ & $6400^{*}$ & Yes \\
145 & 160 & 160 & 180 & 105 & Yes \\
400 & 1522 & 1335 & 716 & 685 & Yes \\
2100 & 1680 & 3250 & 4950 & 4050 & Yes \\
1950 & $200^{*}$ & 325 & 400 & 260 & No \\
520 & 600 & 460 & 240 & 950 & Yes \\
13 & 16 & 10 & 14 & 20 & No \\
4 & 7 & 7 & 10 & 4 & Yes \\
20 & 33 & 34 & 30 & 11 & Yes \\
173 & 311 & 301 & 1259 & 877 & Yes \\
5 & 4 & 6 & 5 & 7 & Yes \\
450 & 1900 & $6400^{*}$ & $6400^{*}$ & $6400^{*}$ & Yes \\
\hline
\end{tabular}

* Censored results. were reviewed. Only subjects fulfilling the entry criteria for the current study and who had paired measurements performed at the same time of day ( \pm 2 hours) were considered. Those whose asthma treatment or occupational exposures were altered between tests, or those who had been exposed to some other agent or procedure which might have influenced airways responsiveness, were excluded. ${ }^{11}$ In addition, results from any subject included in investigation 1 were excluded. Most tests were thereby excluded, leaving 89 paired tests suitable for analysis. For all pairs of tests performed at different intervals a mean refractory index was calculated as the ratio $\mathrm{PD}_{20}$ test $2: \mathrm{PD}_{20}$ test $1 .^{12}$

\section{STATISTICAL ANALYSIS}

Statistical analyses were performed using Minitab (Minitab Inc, Philadelphia, USA) statistical software. The analyses of $\mathrm{PD}_{20}$ and the dose-response slope were performed using logarithmically (base 10) transformed data. Student's $t$ tests and analyses of variance were used in comparisons of baseline $\mathrm{FEV}_{1}, \mathrm{PD}_{20}$, and dose-response slope in investigation 1 , and Student's $t$ tests for analyses of the refractory index in investigation 2 .

\section{Results}

INVESTIGATION 1: SERIAL MEASUREMENTS OF AIRWAYS RESPONSIVENESS OVER THREE DAYS Satisfactory data from all five measurement points were obtained from only 20 of the 24 subjects. Three subjects did not attain a $20 \%$ decrement in $\mathrm{FEV}_{1}$ at the maximum dose of methacholine $(6400 \mu \mathrm{g})$ at some point other than 08:00 hours on day 1 , and one subject attained a greater than $20 \%$ decrement in $\mathrm{FEV}_{1}$ with the first dose of methacholine on one occasion. This subject was tested using the shortened protocol and achieved a $31 \%$ decrement in $\mathrm{FEV}_{1}$ after a first $(400 \mu \mathrm{g})$ dose of methacholine. For these subjects at these time points it was not possible to estimate $\mathrm{PD}_{20}$ using linear interpolation. $\mathrm{A} \mathrm{PD}_{20}$ of $6400 \mu \mathrm{g}$ was therefore assigned to those with an $\mathrm{FEV}_{1}$ decrement $<20 \%$, and a value of $200 \mu \mathrm{g}$ to the subject with a decrement in $\mathrm{FEV}_{1}>20 \%$ after the first dose of methacholine. Results for each subject are shown in table 1 . The dose-response slope could be calculated for all subjects at all time points.

Mean and standard error of $\mathrm{FEV}_{1}$ and log transformed $\mathrm{PD}_{20}$ and dose-response slope results at each measurement point are shown in table 2. There was a significant difference between $\mathrm{FEV}_{1}$ measurements made at different times during day 2 , the 14:00 hours measurements being a mean of $147 \mathrm{ml}$ higher than the 08:00 hours measurements (two way analysis of variance $F_{4,84}=2.859 ; 0.05>\mathrm{p}>0.01$ ). Geometric mean values of $\mathrm{PD}_{20}$ were: 08:00 hours (day 1) $99.5 \mu \mathrm{g}$; 08:00 hours (day 2) $132.4 \mu \mathrm{g} ; 14: 00$ hours (day 2) $160.0 \mu \mathrm{g} ; 20: 00$ hours (day 2) $169.4 \mu \mathrm{g} ; 20: 00$ hours (day 3) $161.4 \mu \mathrm{g}$. Geometric mean values of the doseresponse slope were: 08:00 hours (day 1) 
Table 2 Mean (SE) $F E V_{1}$, geometric mean $P D_{20}$ and geometric mean dose-response slope (DRS) for 24 subjects (investigation 1)

\begin{tabular}{|c|c|c|c|}
\hline Time & Day 1 & Day 2 & Day 3 \\
\hline $\begin{array}{l}\mathrm{FEV}_{1}(\text { lit } \\
08: 00 \\
14: 00 \\
20: 00\end{array}$ & $2 \cdot 732(0 \cdot 189)$ & $\begin{array}{l}2 \cdot 769(0 \cdot 189) \\
2.916(0 \cdot 188) \\
2 \cdot 843(0 \cdot 177)\end{array}$ & $2.869(0.181)$ \\
\hline $\begin{array}{c}\log _{10} P D \\
08: 00 \\
14: 00 \\
20: 00\end{array}$ & $\begin{array}{l}\text { (1.9): } \\
1.998(0 \cdot 161)\end{array}$ & $\begin{array}{l}2 \cdot 122(0 \cdot 176) \\
2 \cdot 204(0 \cdot 199) \\
2 \cdot 229(0 \cdot 201)\end{array}$ & $2 \cdot 208(0 \cdot 203)$ \\
\hline \multicolumn{4}{|c|}{$\begin{array}{c}\log _{10} \text { DRS (\% decrement } \mathrm{FEV}_{1} \text { per } \mu \mathrm{g} \text { methacholine): } \\
08: 00 \quad-0.883(0.174)-0.992(0.201) \\
14: 00 \\
20: 00\end{array}$} \\
\hline
\end{tabular}

$\mathrm{FEV}_{1}=$ forced expiratory volume in one second; $\mathrm{PD}_{20}=$ dose of methacholine causing a $20 \%$ fall in $\mathrm{FEV}_{1}$; DRS = dose-response slope.

$\mathrm{PD}_{20}$ of $6400 \mu \mathrm{g}$ assigned to tests where $\mathrm{FEV}_{1}$ decrement did not reach $20 \%$ and of $200 \mu \mathrm{g}$ where a first dose of $400 \mu \mathrm{g}$ produced a decrement in $\mathrm{FEV}_{1}>20 \%$.

Table 3 Mean ratio (95\% confidence interval) and $p$ values for differences between $P D_{20}$ results for tests performed at each measurement point for 19 subjects studied on consecutive days (investigation 1)

\begin{tabular}{|c|c|c|}
\hline Tests compared & Mean ratio $(95 \%$ CI) & $p$ value \\
\hline $08: 00 / 1 v 08: 00 / 2$ & $1.57(1.19$ to 2.08$)$ & $<0.005$ \\
\hline $08: 00 / 1 \vee 14: 00 / 2$ & $2.01(1.33$ to 3.06$)$ & $<0.005$ \\
\hline $08: 00 / 1 \vee 20: 00 / 2$ & $2.20(1.28$ to 3.79$)$ & $<0.01$ \\
\hline $08: 00 / 1 \vee 20: 00 / 3$ & $2.09(1.27$ to 3.45$)$ & $<0.01$ \\
\hline $08: 00 / 2 \vee 14: 00 / 2$ & $1.28(0.99$ to 1.64$)$ & NS \\
\hline $08: 00 / 2$ v 20:00/2 & $1.40(0.94$ to 2.09$)$ & NS \\
\hline $08: 00 / 2 \vee 20: 00 / 3$ & $1.33(0.87$ to 2.04$)$ & NS \\
\hline $14: 00 / 2 v 20: 00 / 2$ & $1.09(0.77$ to 1.56$)$ & NS \\
\hline $14: 00 / 2$ v 20:00/3 & $1.04(0.71$ to 1.52$)$ & NS \\
\hline $20: 00 / 2$ v 20:00/3 & $0.95(0.69$ to 1.30$)$ & NS \\
\hline
\end{tabular}

Table 4 Mean refractory index $\left(P D_{20}\right.$ test $2 / P D_{20}$ test 1$)$ and $95 \%$ confidence interval for 89 paired methacholine tests performed at different intervals (investigation 2)

\begin{tabular}{lll}
\hline $\begin{array}{l}\text { Interval between } \\
\text { tests (days) }\end{array}$ & $\begin{array}{l}\text { Number of } \\
\text { subjects }\end{array}$ & Refractory index (95\% CI) \\
\hline 1 & 17 & $1.27(1.03$ to $1 \cdot 56)$ \\
$2-4$ & 13 & $0.85(0.68$ to 1.08$)$ \\
$5-6$ & 11 & $0.90(0.63$ to 1.30$)$ \\
7 & 22 & $1.23(0.88$ to $1 \cdot 72)$ \\
$>7$ & 26 & $1.13(0.86$ to 1.48$)$ \\
\hline
\end{tabular}

$-0 \cdot 131 ; 08: 00$ hours (day 2$)-0 \cdot 102 ; 14: 00$ hours (day 2) $-0.091 ; 20: 00$ hours (day 2) $-0.076 ; 20: 00$ hours (day 3) -0.098 . Two way analysis of variance of the $\mathrm{PD}_{20}$ results showed a significant difference between measurements made at different time points $\left(F_{4,92}=\right.$ $2.823,0.05>p>0.01)$, which was confirmed by analysis of the dose-response slope results $\left(F_{4}\right.$, $\left.{ }_{92}=2.995,0.05>\mathrm{p}>0.01\right)$. The largest differences between sequential tests using either measure of airways responsiveness were between the 08:00 hours measurements on days 1 and 2.

As this difference could not have been due to diurnal variation it raised the possibility of prolonged refractoriness to methacholine. Of the 24 subjects 19 had undergone their methacholine tests on consecutive days and five on non-consecutive days. A separate analysis of the results for the group whose tests were performed on consecutive days showed that there was again a significant difference in airways responsiveness between tests, and that removing those subjects not tested on consecutive days had made this difference more

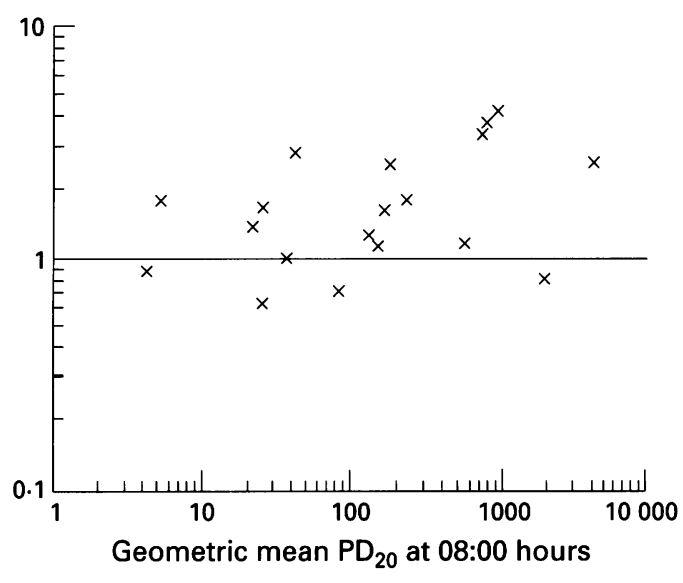

Ratio of $P D_{20}$ at 08:00 hours on day 2 to $P D_{20}$ at 08:00 hours on day 1 against the geomeric mean $P D_{20}$ at 08:00 hours for 19 subjects tested on consecutive days (investigation 1).

apparent (two way analysis of variance for $\mathrm{PD}_{20}$ $F_{4,72}=6.005, \mathrm{p}<0.01$; for dose-response slope $F_{4,72}=4.673, \mathrm{p}<0 \cdot 01$ ). Student's $t$ tests suggested that the only significant differences were those between the 08:00 hours tests on day 1 and all the others (table 3 ). These differences were not related to the $\mathrm{PD}_{20}$ and hence to the dose of methacholine given (figure).

INVESTIGATION 2: REVIEW OF PREVIOUS MEASUREMENTS OF AIRWAYS RESPONSIVENESS Eighty nine paired methacholine tests identified from clinic and research records met the inclusion criteria for the present study. The interval between the paired tests varied from one to 28 days. The mean ratios $\mathrm{PD}_{20}$ test $2: \mathrm{PD}_{20}$ test 1 (refractory index) for first and second tests performed at different intervals are shown in table 4 . The only mean refractory index with a value significantly $>1$ was that obtained at an interval between tests of one day.

\section{Discussion}

This study was designed primarily as an investigation into the importance of diurnal variation in measuring airways responsiveness to methacholine, previous studies having produced conflicting conclusions. ${ }^{1-7}$ One was unable to confirm the existence of diurnal variation in airways responsiveness, ${ }^{7}$ some found a small effect demonstrable only using complex cosinor analysis of repeated measurements, ${ }^{246}$ and others showed an effect but included night time measurements. ${ }^{13-6}$ If diurnal variation does exist, airways responsiveness is likely to be maximal during the night time period, and it is possible that our inability to detect the phenomenon was because we did not make measurements regularly throughout a 24 hour period, in particular at 04:00 hours and 16:00 hours when we might have expected any variation in airways responsiveness to reach its upper and lower limits. Furthermore, although we did detect significant diurnal changes in $\mathrm{FEV}_{1}$, their magnitudes were relatively small. However, we were less interested in the theoretical question of 
what the maximum extent of diurnal variation might be than in the practical question for epidemiological studies of how much it might influence measurements made at different times during normal working hours. We did not detect any significant change in airways responsiveness during normal waking hours, but there was a progressive (albeit non-significant) increase in $\mathrm{PD}_{20}$ (decrease in airways responsiveness) between 08:00 hours and 20:00 hours consistent with the possibility of diurnal variation.

Unexpectedly, there were relatively large differences in $\mathrm{PD}_{20}$ and the dose-response slope between the two 08:00 hours measurements on days 1 and 2, which occurred in the absence of any changes in baseline ventilatory function. For $\mathrm{PD}_{20}$ measurements the magnitude of this difference may have been affected a little by the values given to the censored data, but the dose-response slope data, which were uncensored, also indicated that this was the test interval displaying the greatest mean difference in airways responsiveness. These differences could not have been due to changes in baseline ventilatory function nor to diurnal changes in airways responsiveness, and therefore suggested refractoriness to methacholine. Refractoriness might also have been responsible for the further (though non-significant) sequential increases in $\mathrm{PD}_{20}$ during the second study day. Reanalysis excluding those subjects not tested on consecutive days made the difference between days 1 and 2 at 08:00 hours more apparent, but the differences between all tests on days 2 and 3 remained non-significant.

For the 19 subjects tested on consecutive days it is not clear why a significant degree of refractoriness was not evident also from the 08:00 hours to the 14:00 hours and the 20:00 hours tests on day 2 , although there was some hint of this. It may be that the effect had already reached (or neared) its maximum, or that the mechanism responsible had in some way fatigued. This could also explain why no evidence of refractoriness was seen between the 20:00 hours tests on days 2 and 3.

The possibility of refractoriness was supported by the review of all previous paired data which confirmed that $\mathrm{PD}_{20}$ measurements repeated after 24 hours (but not after longer periods) were significantly higher than the original measurements. This difference was not large, but was of a similar order of magnitude as that found between the 08:00 hours tests on days 1 and 2 in investigation 1 .

Refractoriness to a second methacholine challenge persisting for as long as 24 hours after a first has been described among nonasthmatic subjects with measurable airways responsiveness, but it has not previously been described in asthmatic subjects. ${ }^{13} \mathrm{Re}-$ fractoriness to repeated exposures to the other commonly used bronchoconstrictor agonist, histamine, has been described more frequently, although its existence remains disputed by some and its duration has not been established. ${ }^{1214}$ Refractoriness to other bronchoconstrictor agonists has also been described. ${ }^{15-18}$
Refractoriness to histamine develops within an hour of the first exposure and appears to be related to the dose of histamine administered, with some evidence of a threshold dose (and hence $\mathrm{PD}_{20}$ ) below which it is not observed. ${ }^{12} 14$ The previous demonstration of refractoriness to methacholine in non-asthmatic subjects (who require large doses of bronchoconstrictor agonist to establish a $\mathrm{PD}_{20}$ ) suggests that methacholine refractoriness, as with histamine, might be related to the dose administered. ${ }^{13}$ It could therefore be relevant that our test protocol, which records $\mathrm{FEV}_{1}$ as the mean of the best three of six $\mathrm{FEV}_{1}$ measurements following each dose of methacholine, generally results in higher doses of methacholine being administered than alternative protocols. ${ }^{8}$ In the present study, however, there did not seem to be a threshold below which refractoriness did not occur (figure).

The physiological basis of refractoriness to methacholine is not clear. Several mechanisms have been postulated including depletion of endogenous mediators, neural reflexes, or the release or production of a "protective" substance preventing or ameliorating a further bronchoconstrictor response; and it may be that refractoriness is the result of a number of different interacting mechanisms. ${ }^{151819}$

It seems unlikely that our observations could be due to an artefact or to a learning process in performing spirometric tests. The latter should affect baseline lung function measurements as well as measurements of airways responsiveness, and no significant change in baseline $\mathrm{FEV}_{1}$ was detected between the 08:00 hours measurements on days 1 and 2 . It is conceivable that subjects might be less cautious about taking deep inhalations during the second and subsequent tests. This might lead both to more peripheral (and less effective) deposition of methacholine, and to some functional antagonism of the bronchoconstrictor effect. ${ }^{2021}$ However, such effects should not be more apparent at 24 hours than after longer time intervals (as observed in investigation 2), and we do not believe either explanation to be very convincing. Dilutions of methacholine were prepared daily from a stock solution kept in a dark glass bottle within a refrigerator at $4^{\circ} \mathrm{C}$ so there is no possibility of a systematic deterioration in potency during the course of an individual subject's participation in the study. ${ }^{11}$ We also considered whether we had simply observed the phenomenon of "regression to the mean" following selection of hyperresponsive subjects at entry to the study. However, there was no hint of decreasing responsiveness among the five subjects from investigation 1 , nor the 72 subjects from investigation 2 , who underwent repeated tests on non-consecutive days. For the 72 subjects the mean refractory index (1.07) was close to 1 and not significantly different from it.

The results of this study are of most relevance in the design and interpretation of research involving quickly repeated measurements of airways responsiveness using methacholine. Presumed refractoriness to methacholine, although producing only relatively modest 
changes in $\mathrm{PD}_{20}$ and dose-response slope (of the order of 1.3-1.6 fold at 24 hours), did exert a confounding influence on this study and left us uncertain whether it was the explanation for the progressive increase in $\mathrm{PD}_{20}$ on day 2 or whether there were also diurnal changes in airways responsiveness. Refractoriness might also confound pharmacological studies as it could be interpreted as an effect of treatment. Conversely, refractoriness might reduce the ability to detect increases in airways responsiveness following challenges with aeroallergens or occupational agents. It would therefore seem sensible to ensure that the possibility of prolonged refractoriness to methacholine is taken into account in analyses, or that an appropriate minimum period of 48 hours is allowed between sequential tests.

We cannot exclude the possibility of diurnal variation in measurements of airways responsiveness to methacholine, particularly in subjects showing a prominent diurnal variation in ventilatory function. However, we believe that any change during normal waking (and working) hours is not likely to exert an important confounding effect in epidemiological investigations, and that diurnal variation is of less consequence than refractoriness.

1 de Vries K, Goei JT, Booy-Noord H, Orie NGM. Changes during 24 hours in the lung function and histamine hyperreactivity of the bronchial tree in asthmatic and bronchitic patients. Int Arch Allergy 1962;20:93-101.

2 Rachiele A, Malo JL, Cartier A, Pineau I, Ghezzo H, Martin $R R$. Circadian variations of airway response to histamine in asthmatic subjects. Bull Eur Physiopathol Respir 1983; 19:465-9.

3 van Aalderen WMC, Postma DA, Koeter GH, Knol K Circadian changes in bronchial responsiveness and airflow obstruction in asthmatic children. Thorax 1989;44:803-7.

4 Dreher D, Koller EA. Circadian rhythms of specific airway conductance and bronchial reactivity to histamine: the effects of parasympathetic blockade. Eur Respir $\mathcal{F} 1990 ; 3$ : 414-20.

5 Bonnet R, Jorres R, Heitmann U, Magnussen H. Circadian rhythm in airway responsiveness and airway tone in patients with mild asthma. F Appl Physiol 1991;71:1598605.

6 Kondo S, Abe K. Priority of peak circadian variation of bronchial responsiveness to the trough of circadian variation of bronchial caliber in asthmatic children. Chest 1991;100:640-3.

7 Bundgaard A, Andersen KK. Histamine challenge in asthmatics at 07:00, 12:00, 17:00 and 22:00 h. Eur $\mathcal{F}$ Respir Dis 1986;68(Suppl 143):14-17

8 Beach JR, Young CL, Avery AJ, Stenton SC, Dennis JH, Walters EH, et al. Measurement of airway responsiveness to methacholine: relative importance of the precision of drug delivery and the method of assessing response. Thorax 1993;48:239-43.

9 Hendrick DJ, Fabbri LM, Hughes JM, Banks DE, Barkman HW, Connolly MJ, et al. Modification of the methacholine inhalation test and its epidemiologic use in polyurethane workers. Am Rev Respir Dis 1986;133:600-4.

10 O'Conner G, Sparrow D, Taylor D, Segal M, Weiss S. Analysis of dose response curves to methacholine. An approach suitable for population studies. Am Rev Respir Dis 1987;136:1412-17.

11 Sterk PJ, Fabbri LM, Quanjer Ph H, Cockroft DW, O'Byrne PM, Anderson SD, et al. Airway responsiveness. Standardised challenge testing with pharmacological, physical and sensitising stimuli in adults. Report working party and sensitising stimuli in adults. Report working party munity for Steel and Coal. Official statement of the Euromunity for Steel and Coal. Official statement of the European Resp.

12 Connolly MJ, Stenton SC, Avery AJ, Walters EH, Hendrick DJ. Refractory period following bronchoconstriction provoked by histamine in asthmatic subjects. Thorax 1989 44:146-50.

13 Beckett WS, McDonnell III WF, Wong ND. Tolerance to methacholine inhalation challenge in nonasthmatic submethacholine inhalation challenge in nonast

14 Polosa R, Finnerty JP, Holgate ST. Tachyphylaxis to inhaled histamine in asthma: its significance and relationship to basal airway responsiveness. $\mathcal{F}$ Allergy Clin Immunol 1990; 86:265-71.

15 Mattoli S, Foresi A, Corbo GM, Valente S, Culla G, Ciappi G. Refractory period to ultrasonic mist of distilled water: relationship to methacholine responsiveness, atopic status, and clinical characteristics. Ann Allergy 1987;58:134-40.

16 Belcher NG, Rees PJ, Clark TJH, Lee TH. A comparison of the refractory periods induced by hypertonic airway challenge and exercise in bronchial asthma. Am Rev Respir challenge and exercise in

17 Rosenthal RR, Laube BL, Hood DB, Norman PS. Analysis of refractory period after exercise and eucapnic voluntary hyperventilation challenge. Am Rev Respir Dis 1990;141: 368-72.

18 Pavord I, Lazarowicz H, Inchley D, Baldwin D, Knox A, Tattersfield A. Cross refractoriness between sodium metabisulphite and exercise induced asthma. Thorax 1994; 49:245-9.

19 Hahn AG, Nogrady SG, Tumilty McA, Lawrence SR Morton AR. Histamine reactivity during the refractory period after exercise-induced asthma. Thorax 1984;39: 919-23.

20 Orehek J, Nicoli MM, Delpierre S, Beaupre A. Influence of the previous deep inspiration on the spirometric measurement of provoked bronchoconstriction in asthma. Am Rev Respir Dis 1981;123:269-72.

21 Wheatley JR, Pare PD, Engel LA. Reversibility of induced bronchoconstriction by deep inspiration in asthmatic and normal subjects. Eur Respir f 1989;2:331-9. 